Disponível em

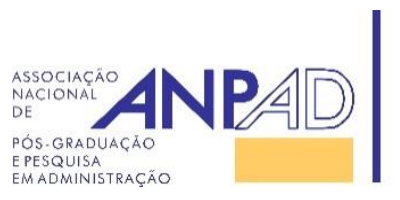

http://www.anpad.org.br/rac

RAC, Rio de Janeiro, v. 21, n. 1, art. 2, pp. 19-40, Jan./Fev. 2017

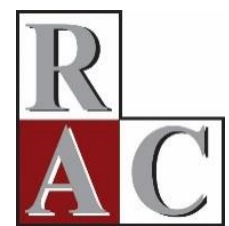

$(c)$ EY

\title{
Jogos de Empresas: Abordagens ao Fenômeno, Perspectivas Teóricas e Metodológicas
}

Business Games: Approaches to the Phenomenon, Theoretical and Methodological Perspectives

Valdete de Oliveira Mrtvi $^{1}$ Fernando Kaname Westphal ${ }^{1}$

Rodrigo Bandeira-de-Mello ${ }^{2}$

Paulo Roberto Feldmann ${ }^{3}$

Universidade Estadual de Londrina ${ }^{1}$

Fundação Getulio Vargas ${ }^{2}$

Universidade de São Paulo ${ }^{3}$

Artigo recebido em 15.08.2015. Última versão recebida em 01.02.2016. Aprovado em 07.02.2016. Publicado online em 31.08.2016. 


\title{
Resumo
}

Os jogos de empresas, além de utilizados no processo de ensino, também são explorados como ferramentas de pesquisa, conforme Gentry, Tice, Robertson e Gentry (1984). Este estudo, de natureza qualitativa e quantitativa, descritivo quanto aos objetivos, e caracterizado como revisão integrativa no tocante ao método, trata do seguinte problema de pesquisa: Qual o perfil da pesquisa acadêmica brasileira sobre jogos de empresas considerando a abordagem ao fenômeno e as perspectivas teóricas e metodológicas? Foram analisados 226 artigos extraídos de periódicos online, do indexador Spell e anais de eventos. Como resultado, é proposta uma tipologia para classificação das pesquisas em jogos de empresas, considerando a abordagem ao fenômeno de pesquisa e os eixos temáticos. Constatou-se a predominância de estudos que têm as simulações como objeto de pesquisa - SCOPE $(77,9 \%)$-, enquanto $22,1 \%$ utilizam os jogos de empresas como ambiente de pesquisa - SCAPE. Verificou-se que 38,5\% dos estudos não mencionam quaisquer das classificações metodológicas analisadas. Discute-se algumas fragilidades metodológicas identificadas nas pesquisas da área. Apesar de as classificações não serem determinantes para a qualidade de um estudo, representam importante indicador da consistência do processo de reflexão e da coesão da investigação.

Palavras-chave: jogo de empresas; simulação; estratégia de pesquisa; tipologia de pesquisa.

\begin{abstract}
In addition to being used in the teaching process, Business Games are used as research tools, as pointed out by Gentry, Tice, Robertson and Gentry (1984). This qualitative, quantitative and descriptive study is characterized as an integrative review regarding methodology, and deals with the following research problem: what are the characteristics of Brazilian academic research articles on business games, considering their approach to the phenomenon, theoretical background and methodological aspects? A total of 226 articles published in online scientific journals, as well as the Spell indexer and in conferences were analyzed. Results produced a proposed typology for the classification of research in business games, considering: (a) research approach to the phenomenon; and (b) theoretical themes. A predominance of studies was observed to focus on simulations as research objects - SARO (77.9\%), while $22.1 \%$ use business simulations as a research environment - SARE. Among the studies, $38.5 \%$ were found to not mention any of the methodological classifications analyzed. The paper discusses some methodological weaknesses identified in the research area. Although classification is not instrumental in determining the quality of a study, it is evidence of consistency in the reflection process and of cohesion in the research.
\end{abstract}

Key words: business game; simulation game; research design; research typology. 


\section{Introdução}

Tradicionalmente, o fenômeno jogo de empresas é estudado considerando suas características didático-pedagógicas (Motta, Melo, \& Paixão, 2012), sua eficiência e suas formas de desenvolvimento (Rosas \& Sauaia, 2009), diante da importância deste ferramental no contexto de ensino-aprendizagem nos cursos da área de negócios. Apesar de essa vertente de pesquisa ser predominante, a exploração do ferramental fornecido pelos jogos de empresas e simuladores como instrumento de pesquisa é uma possibilidade discutida por diversos autores (Bass, 1964; Cohen \& Rhenman, 1961; Gentry, Tice, Robertson, \& Gentry, 1984; Keys \& Wolfe, 1990; Larréché, 1987; Sauaia, 2013), o que leva a uma distinção no que se refere à abordagem ao fenômeno desses dois tipos de pesquisa.

Segundo Richardson (2012, p. 57) "o fenômeno tem características próprias e ocupa lugar no tempo ... [, ele] existe, tem essência e é objeto do conhecimento científico". Assim, segundo o autor, o conceito de fenômeno de pesquisa permite melhor delimitação do estudo, na medida em que vincula o pesquisador ao objeto da pesquisa.

$\mathrm{Na}$ abordagem mais tradicional, o fenômeno em estudo são os jogos de empresas e simuladores, ou seja, o seu desenvolvimento, a sua eficiência como instrumento de aprendizagem e outros aspectos são estudados, particularmente no contexto de ensino-aprendizagem. Na segunda abordagem, diferentemente da anterior, o fenômeno em estudo não é circunscrito ao jogo de empresas ou simulador, ou seja, tais sistemas funcionam como ferramental para recriar circunstâncias que possibilitam aos pesquisadores a observação e a coleta de dados acerca do fenômeno pesquisado, que podem envolver aspectos comportamentais, ou, ainda, a tomada de decisão.

A divisão dos estudos realizados na área, considerando os que investigam o fenômeno jogo de empresas e aqueles que o utilizam como laboratório de pesquisa, foi apontada por Keys e Wolfe (1990). Mais recentemente, Oliveira, Sauaia, Garcia e Moreira (2010) também ressaltam essa divisão, indicando o aumento do interesse dos pesquisadores em investigar os jogos de empresas, seja como objeto de pesquisa, seja como ambiente experimental controlado. Para esses autores, ainda é reduzido o número de pesquisas que utilizam o jogo de empresas como instrumento de pesquisa. Paixão, Bruni e Carvalho (2007) também apontam que "poucos são os trabalhos envolvendo pesquisa empírica sobre assunto diverso, que utilize os jogos de empresas como experimento, criando os verdadeiros laboratórios de gestão empresarial" (p. 8). Nesse sentido, o presente estudo foi conduzido considerando a necessidade de analisar em que medida os simuladores e jogos de empresas são adotados como instrumentos de pesquisa no Brasil, além de avaliar as características metodológicas dessas pesquisas.

Ademais, percebe-se que diferentes temáticas e perspectivas teóricas têm sido adotadas, abrangendo tanto a área de operações como as áreas de economia e tomada de decisão.

Diante do exposto, a fim de investigar mais profundamente como os jogos de empresas são considerados nas pesquisas brasileiras, definiu-se o problema: Qual o perfil da pesquisa acadêmica brasileira sobre jogos de empresas considerando a abordagem ao fenômeno e as perspectivas teóricas e metodológicas? Para responder a este problema de pesquisa, identificou-se a necessidade do desenvolvimento de uma tipologia dos estudos em jogos de empresas no que se refere à abordagem ao fenômeno e às perspectivas teóricas. Essa tipologia, além de ser uma importante etapa para se atingir o objetivo geral, constitui uma das principais contribuições deste estudo.

O objetivo geral do estudo foi analisar a pesquisa acadêmica brasileira sobre jogos de empresas considerando a abordagem ao fenômeno e as perspectivas teóricas e metodológicas. Assim, os seguintes objetivos específicos foram determinados: (a) identificar a frequência e a natureza das publicações na área de jogos de empresas considerando as abordagens ao fenômeno; (b) elaborar uma tipologia de classificação das diferentes perspectivas teóricas, considerando as temáticas e as abordagens ao fenômeno; e (c) analisar a metodologia empregada pelas pesquisas na área a partir das classificações utilizadas, considerando a abordagem metodológica, o método e o tipo de pesquisa quanto ao objetivo. 


\section{Revisão Bibliográfica}

A origem dos jogos de empresas, apesar ser associada aos jogos de tabuleiro e de guerra, tem como predecessoras diretas três simulações: (a) em 1932, na Europa, Mary Birshstein modelou o processo de produção de máquinas de escrever (Gagnon, 1987 como citado em Faria, Hutchinson, Wellington, \& Gold, 2009); (b) Monopologs, concebida, em 1955, pela RAND Corporation, que simulava os sistemas de abastecimento da força aérea americana (Jackson, 1959 como citado em Faria et al., 2009); e (c) por fim, em 1956, foi desenvolvida pela American Management Association (AMA) a Top Management Decision Simulation, que se tornou uma das simulações mais conhecidas (Hodgetts, 1970 como citado em Faria et al., 2009).

Dessa forma, os jogos de empresas são definidos como "exercícios de tomada de decisão em que equipes competem em busca de satisfazer objetivos específicos" (Babb, Leslie, \& Van Slyke, 1966, p. 466). Os jogos de empresas funcionam como um ambiente vivencial simulado, definido por Keys e Wolfe (1990, p. 308) como "uma situação simplificada e restrita que contém suficiente verossimilhança ou ilusão de realidade para induzir, nos participantes do exercício, respostas como as do mundo real".

Muitos jogos de empresas utilizam simuladores computadorizados, ou seja, sistemas em servidores locais ou na internet que processam as decisões e geram resultados aos participantes, enquanto outros jogos de empresas empregam recursos mais simples como tabuleiros, cartas e fichas. Sauvé, Renaud, Kaufman e Marquis (2007) distinguem jogos e simulações. O jogo consiste em uma "ficção, fantasia ou situação artificial na qual jogadores, colocados em conflito entre si ou contra outras forças, são governados por regras que estruturam suas ações para alcançar objetivos de aprendizado, bem como uma meta determinada pelo jogo" (Sauvé, Renaud, Kaufman, \& Marquis, 2007, p. 251). Já a simulação, no contexto de aprendizagem, é definida, por Sauvé et al. (2007, p. 258), como "uma representação simplificada, dinâmica e precisa de uma realidade definida como um sistema". WrightMaley (2015) pontua que, no contexto das ciências sociais, as simulações: (a) refletem a realidade de maneira estruturada e de forma limitada; (b) ilustram eventos dinâmicos, processos ou fenômenos; (c) incorporam os aprendizes em papéis ativos por meio dos quais o fenômeno se revela; e (d) são mediadas pedagogicamente.

Além das distinções conceituais entre jogo de empresas e simulação, a variedade de jogos, formatos e modos de desenvolvimento e aplicações de tecnologia suscitou discussão acerca de suas diferentes classificações e propostas de tipologias. Cabe destacar que essa área se beneficiou em grande parte da evolução tecnológica ocorrida nos últimos quarenta anos, conforme evidencia a revisão efetuada por Faria, Hutchinson, Wellington e Gold (2009), fato que alavancou a utilização e disseminação dos jogos de empresas e simuladores. Além de possuírem grande potencial como ferramenta de ensino, também são explorados há algum tempo como instrumentos de pesquisa, de acordo com Gentry, Tice, Robertson e Gentry (1984). A pesquisa por meio de jogos e simulações, principalmente quanto ao seu potencial e limitações, foi discutida em diversos estudos (Bass, 1964; Cohen \& Rhenman, 1961; Gentry et al., 1984; Keys \& Wolfe, 1990; Larréché, 1987; Sauaia, 2013). Mais recentemente, Dieguez-Barreiro, Gonzáles-Benito, Galende e Kondo (2011) retomam a discussão acerca dos jogos de empresas como instrumental na pesquisa em administração.

Diante desse contexto, a revisão bibliográfica é dividida em duas partes. Na primeira, são apresentadas algumas das tipologias existentes em jogos de empresas. Na sequência, são expostas revisões da literatura na área de jogos e simulações, bem como são definidos os limites entre o presente estudo e pesquisas anteriores.

\section{Tipologias na área de jogos de empresa}

As tipologias possuem importante papel por permitirem ao pesquisador a ordenação do conhecimento existente em determinada área. Possuem também a função de agregação, que possibilita partir de um nível inferior de abstração para um mais elevado, além de viabilizar o acúmulo de conhecimento (Bertero, 1981). 
No campo dos jogos de empresas, o processo de tipificação possibilitou um maior entendimento da natureza desse fenômeno e dos diversos elementos que o compõem. Biggs (1990), em revisão de uma série de estudos da década de 1960 e 1970, ao discutir a natureza dos jogos de empresas, classifica-os em: (a) jogos totais e jogos funcionais, considerando a amplitude; (b) competitivos ou não competitivos, considerando a interferência ou não das decisões de uma equipe no resultado de outras; (c) interativos e não interativos; (d) genérico ou de indústria específica; (e) individuais ou de equipe; (f) deterministas ou estocásticos; e (g) em relação ao tipo de computador, ao grau de complexidade e ao período jogado.

No Brasil, Lacruz (2004) realiza a síntese de algumas tipologias de jogos de empresas (Tabela 1), não abrangendo, porém, classificações das áreas de pesquisa em jogos.

Tabela 1

Classificações dos Jogos de Empresa

\begin{tabular}{|c|c|c|}
\hline Autor & Critério de Classificação & Agrupamento \\
\hline \multirow[t]{5}{*}{ Tanabe (1977) } & Quanto ao meio de apuração & Manuais ou computadorizados. \\
\hline & Quanto às áreas funcionais & Jogos gerais ou jogos funcionais. \\
\hline & $\begin{array}{l}\text { Quanto à interação entre as } \\
\text { equipes }\end{array}$ & Interativos ou não interativos. \\
\hline & Quanto ao setor da economia & Industrial, comercial, financeiro, serviços. \\
\hline & Quanto ao tempo de resposta & Tempo real, por correspondência, processamento remoto. \\
\hline Wilhelm (1997) & $\begin{array}{l}\text { Quanto à tecnologia } \\
\text { empregada }\end{array}$ & $\begin{array}{l}\text { Subdivido em jogos de } 1^{\mathrm{a}}, 2^{\mathrm{a}} \text { e } 3^{\mathrm{a}} \text { geração considerando o } \\
\text { tipo de tecnologia empregada (como mainframes, } \\
\text { microcomputadores e sistemas interativos). }\end{array}$ \\
\hline Gramigna (1993) & $\begin{array}{l}\text { Quanto às habilidades } \\
\text { desenvolvidas (espectro e } \\
\text { direcionamento) }\end{array}$ & $\begin{array}{l}\text { Jogos voltados para o desenvolvimento pessoal, gerencial, } \\
\text { ou que enfatizam habilidades técnicas de mercado. }\end{array}$ \\
\hline \multirow[t]{3}{*}{ Vicente (2001) } & Quanto aos objetivos & $\begin{array}{l}\text { Edutainment: jogos lúdicos com objetivos de aprendizagem. } \\
\text { Analíticos: visam estudar os aspectos de um determinado } \\
\text { negócio. }\end{array}$ \\
\hline & Quanto à forma & Jogos de computador, tabuleiro, dramatização, entre outros. \\
\hline & Quanto aos modelos & $\begin{array}{l}\text { Modelos estatísticos, probabilísticos, determinísticos, } \\
\text { sistemas caóticos. }\end{array}$ \\
\hline Motomura (1980) & $\begin{array}{l}\text { Quanto à natureza básica do } \\
\text { jogo }\end{array}$ & $\begin{array}{l}\text { Jogos sistêmicos (funcionamento do sistema), humanos } \\
\text { (variáveis humanas presentes nas negociações), ou mistos } \\
\text { (componentes sistêmicos e humanos). }\end{array}$ \\
\hline \multirow[t]{4}{*}{ Bernard (1993) } & $\begin{array}{l}\text { Quanto à abordagem do } \\
\text { problema gerencial }\end{array}$ & $\begin{array}{l}\text { Jogos gerais (focam principais áreas da organização) ou } \\
\text { jogos funcionais (focalizam áreas específicas). }\end{array}$ \\
\hline & $\begin{array}{l}\text { Quanto à interação entre } \\
\text { equipes }\end{array}$ & $\begin{array}{l}\text { Interativo (decisões das empresas influenciam e são } \\
\text { influenciadas pelas demais decisões), sendo que o contrário } \\
\text { ocorre com jogos classificados como isolados. }\end{array}$ \\
\hline & Quanto aos objetivos & $\begin{array}{l}\text { Individual ou em grupo, dependendo do tipo de habilidade } \\
\text { a ser desenvolvida. }\end{array}$ \\
\hline & $\begin{array}{l}\text { Quanto ao tipo de variáveis } \\
\text { envolvidas }\end{array}$ & Determinístico ou estocástico. \\
\hline
\end{tabular}

Quanto à dinâmica

Estático ou dinâmico, dependendo do comportamento das variáveis.

Nota. Fonte: Adaptado de Lacruz, A. J. (2004). Jogos de empresas: considerações teóricas. Caderno de Pesquisas em Administração, 11(4), 93-109. Recuperado de http://www.unifal.com.br/Bibliotecas/Artigos_Cientificos/JOGOS\%20DE\%20EMPRESAS\%20CONSIDERA\%C3\%87\%C3 $\% 95 E S \% 20 T E O R I C A S . p d f$.

RAC, Rio de Janeiro, v. 21, n. 1, art. 2, pp. 19-40, Jan./Fev. 2017

WWw.anpad.org.br/rac $(\mathrm{oc}) \mathbf{E}$ 
No âmbito dos jogos e da área da educação, Greco, Baldissin e Nonino (2013) propuseram uma ampla taxonomia com o objetivo de facilitar a comparação de ferramentas educacionais baseadas em jogos, focando os componentes associados ao aprendizado. Essa taxonomia foi ainda expandida por Blažič e Blažič (2015), que incorporaram a dimensão aprendizagem com o propósito de classificar a forma com que os jogos atingem os objetivos educacionais.

Diante dessas contribuições, percebe-se que as tipologias apresentadas representaram um passo importante para a própria definição do conceito de jogo de empresas, além de permitir que desenvolvedores e aplicadores compreendessem as diversas dimensões envolvidas em um jogo, não só as pertinentes ao simulador e aos aspectos computacionais, como também a própria dinâmica do processo de aprendizagem. Apesar de essas tipologias se concentrarem nos jogos de empresas e não abordarem classificações dos tipos de pesquisas, ou do campo em si, alguns autores realizaram importantes contribuições nesse sentido.

Cohen e Rhenman (1961) relatam o interesse dos desenvolvedores na utilização de jogos como ferramenta de pesquisa. Discutem, também, a utilidade dos jogos de empresas como laboratório de ciências sociais e estabelecem a seguinte classificação: (a) jogos de empresas para a solução de problemas gerenciais; (b) pesquisa em economia; (c) pesquisa em teoria organizacional; e (d) pesquisa em psicologia.

Larréché (1987), por sua vez, agrupa as pesquisas realizadas na área de jogos e simulações em três tópicos principais: (a) simulações como ferramentas de ensino; (b) tomada de decisão e manipulação de informações; e (c) organização, liderança e traços pessoais. $\mathrm{O}$ autor apresenta, ainda, o conceito de laboratório de ambiente simulado, que permite "prover um contexto geral para atividades educacionais e de pesquisa" (Larréché, 1987, p. 565).

Entre as classificações revisadas no presente trabalho, Keys e Wolfe (1990) realizaram a categorização mais ampla ao considerarem, também, estudos que utilizam os jogos de empresas como laboratórios de pesquisa (research laboratories). Apesar de autores precursores já terem identificado o potencial deste ferramental, Keys e Wolfe (1990) identificaram e subdividiram as pesquisas da área em dois grupos (Tabela 2), sendo o primeiro composto por estudos que delimitam os jogos como fenômeno, enquanto o outro comporta investigações que utilizam as simulações como laboratório experimental.

Tabela 2

Classificações das Pesquisas por Tipo de Estudo e Área de Aplicações dos Experimentos

\begin{tabular}{|c|c|}
\hline Tipos de estudos sobre jogos de empresas & $\begin{array}{l}\text { Jogos de empresas como laboratórios de pesquisa } \\
\text { (áreas) }\end{array}$ \\
\hline $\begin{array}{l}\text { 1. Aprendizagem comparada: cursos de gestão estratégica } \\
\text { versus casos }\end{array}$ & $\begin{array}{l}\text { 1. Sistemas de suporte à decisão e de processamento de } \\
\text { informações }\end{array}$ \\
\hline $\begin{array}{l}\text { 2. Aprendizagem comparada: outros cursos versus } \\
\text { casos }\end{array}$ & 2. Pesquisa em administração estratégica \\
\hline 3. Validade Externa & 3. Comportamento do grupo e tomada de decisão \\
\hline 4. Complexidade do jogo & 4. Pesquisa organizacional \\
\hline 5. Validade do algoritmo & 5. Pesquisa em liderança \\
\hline \multicolumn{2}{|l|}{ 6. Escopo funcional } \\
\hline \multicolumn{2}{|l|}{ 7. Atitudes e níveis de desempenho do estudante } \\
\hline \multicolumn{2}{|l|}{ 8. Coesão e composição da equipe } \\
\hline 9. Tamanho da equipe & \\
\hline
\end{tabular}




\section{Tabela 2 (continuação)}

\begin{tabular}{ll}
\hline Tipos de estudos sobre jogos de empresas & $\begin{array}{l}\text { Jogos de empresas como laboratórios de pesquisa } \\
\text { (áreas) }\end{array}$ \\
\hline
\end{tabular}

10 Fases de aprendizado

11. Peso das avaliações

12. Papel do instrutor/animador do jogo

13. Instrumentos e materiais complementares

Nota. Fonte: Adaptado de Keys, B., \& Wolfe, J. (1990). The role of management games and simulations in education and research. Journal of Management, 16(2), 307-336. Recuperado de http://jom.sagepub.com/content/16/2/307.abstract. http://dx.doi.org/10.1177/014920639001600205

Nesse contexto, uma das etapas desta pesquisa envolveu a proposição de uma classificação dos estudos realizados no país, explorando particularmente a dicotomia existente entre esses dois tipos de investigação.

\section{Pesquisas sobre publicações na área de jogos de empresas}

A área de pesquisa envolvendo simulações e jogos de empresas foi foco de estudo de diversos autores no Brasil (Barçante \& Beltrão, 2013; Motta, Melo, Oliveira, Quintella, \& Garcia, 2011; Paixão, Bruni, \& Carvalho, 2007). Tais estudos representaram esforços de análise da produção acadêmica na área, tanto no Brasil como internacionalmente, conforme observado no artigo de Motta, Melo, Oliveira, Quintella e Garcia (2011). À exceção deste, tais estudos focaram predominantemente artigos publicados em anais de eventos, que constituem importante meio de comunicação científica para pesquisadores da área.

Nas revisões anteriormente realizadas, o volume de publicações constituiu indicador mais abordado e analisado, considerando diferentes perspectivas de comparações (como temporal e por tipo de publicação). Tal ênfase deriva do próprio método adotado para essas pesquisas, o bibliométrico e variações (research profiling e perfil da pesquisa), cuja proposta é quantificar a produção, preferencialmente por meio de ferramentas e recursos automatizados para contagem de termos e palavras de interesse. Ao mesmo tempo que tais recursos permitem abranger grande volume de publicações e possuem elevado nível de confiabilidade devido à automação, as análises de aspectos qualitativos e metodológicos podem ser limitadas, na medida em que tais métodos dispensam a leitura individual dos trabalhos. O crescimento do número de publicações na área de jogos de empresas no Brasil foi constatado por Paixão et al. (2007) e Barçante e Beltrão (2013). Motta et al. (2011), por sua vez, criticaram a relativa imaturidade do campo em virtude da concentração das publicações em poucas redes de coautoria, resultados que se aproximaram dos achados de Bragge, Thavikulwat e Töyli (2010), que apontaram para a existência de 7 a 11 clusters de autores, considerando os trabalhos referenciados.

No âmbito internacional, Bragge et al. (2010) publicaram uma das revisões mais abrangentes da área de simulação em que analisaram 40 anos de publicações do periódico Simulation \& Gaming, sistematizando importantes dados com relação à produtividade, à coautoria entre os pesquisadores, aos periódicos e aos autores mais referenciados, bem como aos termos mais utilizados em títulos e campos destinados à descrição dos trabalhos.

Mais recentemente, Halpin (2013), em revisão da pesquisa sobre simulações, localizou 20 artigos publicados no periódico Academy of Management Journal (AMJ) até meados de 2012. A autora classificou-os considerando as variáveis abordadas pelos estudos, identificando cinco construtos: desempenho, processos de grupo/tomada de decisão, definição de objetivos, sistemas e coleta de informações e estratégia.

Considerando o cenário retratado, esta pesquisa foi conduzida com o intuito de ampliar a discussão iniciada pelos estudos relacionados, na medida em que: (a) considera, para definição da 
amostra de artigos, trabalhos publicados tanto em anais quanto em periódicos; (b) adota uma metodologia de pesquisa que prevê a leitura de todos os artigos selecionados; e (c) propõe uma tipologia de classificação das pesquisas realizadas na área de jogos de empresas considerando a abordagem ao fenômeno e os eixos temáticos.

\section{Procedimentos Metodológicos}

Este estudo, de natureza qualitativa e quantitativa, e descritivo quanto aos objetivos, foi caracterizado como revisão integrativa de escopo limitado por abordar temáticas específicas de um determinado estudo (Tabela 3).

Tabela 3

\section{Processo de Revisão Integrativa Conduzido na Presente Pesquisa}

\begin{tabular}{ll}
\hline Passos da revisão integrativa & Síntese das ações conduzidas na pesquisa \\
\hline $\begin{array}{l}\text { 1. Identificação do tema } \\
\text { seleção da questão de pesquisa }\end{array}$ & $\begin{array}{l}\text { Definição dos elementos para determinação do perfil da pesquisa acadêmica } \\
\text { em jogos: abordagem ao fenômeno, eixos teóricos e metodologia; }\end{array}$ \\
& $\begin{array}{l}\text { Definição das bases mais relevantes para a área (anais de eventos, periódicos } \\
\text { e indexador de periódicos). }\end{array}$
\end{tabular}

2. Estabelecimento dos critérios . Definição das palavras-chave para a busca dos estudos. de inclusão e exclusão
3. Identificação dos estudos pré- . Identificação de 268 estudos;
selecionados e selecionados . Leitura e aplicação dos critérios que determinaram se os estudos tratavam de simuladores e/ou jogos de empresas;
. Seleção de 51 estudos em periódicos e 190 em anais de eventos.

4. Categorização dos estudos . Desenvolvimento de uma tipologia para avaliação dos estudos, considerando selecionados a abordagem ao fenômeno e as teorias adotadas;

. Organização das informações dos estudos em planilha eletrônica e análise.

5. Análise e interpretação dos . Discussão dos resultados agregados envolvendo abordagem ao fenômeno, resultados eixos teóricos e características metodológicas.

\begin{tabular}{|c|c|}
\hline $\begin{array}{l}\text { 6. Apresentação da } \\
\text { revisão/síntese do conhecimento }\end{array}$ & $\begin{array}{l}\text { Elaboração do artigo com uso de gráficos e tabelas que sintetizam as } \\
\text { constatações da pesquisa. }\end{array}$ \\
\hline
\end{tabular}

Nota. Fonte: Adaptado de Botelho, L. L. R., Cunha, C. C. de. A., \& Macedo, M. (2011). O método da revisão integrativa nos estudos organizacionais. Gestão $e$ Sociedade, 5(11), 121-36. Recuperado de http://www.gestaoesociedade.org/gestaoesociedade/article/download/1220/906. http://dx.doi.org/10.21171/ges.v5i11.1220

Botelho, Cunha e Macedo (2011) definem o processo para a condução de uma revisão integrativa cujas etapas são listadas na Tabela 3, juntamente com uma síntese das ações correspondentes que foram executadas nesta pesquisa.

Diante do propósito de analisar o perfil da pesquisa em jogos de empresas considerando as abordagens ao fenômeno, os eixos teóricos e os aspectos metodológicos, a revisão integrativa é pertinente, uma vez que, conforme Botelho et al. (2011), o propósito deste tipo de pesquisa é revisar métodos, teorias e/ou estudos empíricos sobre um tópico particular.

Para a definição das respectivas bases pesquisadas, primeiramente foi efetuado um levantamento exploratório em periódicos on-line, no indexador Spell e em anais de eventos relacionados à área de gestão. Esta busca levou à identificação de quatro eventos que se destacam como receptores de estudos relacionados aos simuladores de gestão empresarial, que são (Tabela 4): Encontro Nacional dos 
Programas de Pós-Graduação em Administração (ENANPAD), Encontro Nacional de Engenharia de Produção (ENEGEP), Seminários em Administração (SEMEAD) e Simpósio de Engenharia de Produção (SIMPEP).

Tabela 4

Número de Artigos Identificados em Anais de Eventos

\begin{tabular}{lccccc}
\hline & ENANPAD & ENEGEP & SEMEAD & SIMPEP & Total \\
\hline Número de artigos & 34 & 61 & 62 & 33 & 190 \\
\hline
\end{tabular}

Nota. Fonte: Dados da pesquisa.

A busca foi estendida para os periódicos após a análise das referências bibliográficas dos estudos selecionados nos anais, o que levou à inclusão das seguintes revistas (Tabela 5): Revista de Administração Contemporânea (RAC), Revista Eletrônica de Estratégia e Negócios (REEN), Revista de Gestão da USP (REGEUSP), Revista Eletrônica de Administração (REAd), Revista de Negócios, Revista de Administração de Empresas (RAE), Revista de Administração da Universidade de São Paulo (RAUSP) e Administração: Ensino e Pesquisa (RAEP).

Tabela 5

Número de Artigos Identificados em Periódicos

\begin{tabular}{lcccccccccc}
\hline & RAC $^{\mathrm{a}}$ & REEN & REGEUSP & REAd & $\begin{array}{c}\text { Revista de } \\
\text { Negócios }\end{array}$ & RAE & RAUSP & RAEP & Outros $^{\mathrm{b}}$ & Total $^{\text {Reta }}$ \\
\hline $\begin{array}{l}\text { Número de } \\
\text { artigos }\end{array}$ & 5 & 5 & 4 & 4 & 4 & 3 & 3 & 3 & 20 & 51 \\
\hline
\end{tabular}

Nota. Fonte: Dados da pesquisa.

a Inclui RAC-Eletrônica; Inclui a revista BAR e 17 periódicos indexados pelo Spell (ADM.MADE; RECADM; E\&G; ReA UFSM; Contextus; O\&S; BAR; CVT; InternexT; PG\&C; Reuna; RIAE; Alcance; RBGN; Revista Contabilidade \& Finanças - USP; RMPE; Revista de Contabilidade do Mestrado em Ciências Contábeis da UERJ; Revista Pensamento Contemporâneo em Administração).

Assim, do total de 268 artigos pré-selecionados, 241 puderam ser classificados como da área de jogos de empresas e 226 foram analisados neste estudo, uma vez que 14 foram publicados tanto em anais de eventos como em periódicos; portanto, foram consideradas somente publicações em periódicos. Além disso, um trabalho foi excluído devido à impossibilidade de acesso ao texto na íntegra.

Registra-se também que 27 artigos (dos 268 pré-selecionados) foram excluídos por empregarem os termos pesquisados fora do contexto deste estudo. Neste caso, enquadram-se aqueles que empregaram o termo jogo como sinônimo de disputa ou embuste, no contexto de pesquisas sobre estratégia e poder, além daqueles que apresentaram o termo simulação como técnica de cálculo com intuito preditivo.

A seleção dos artigos para compor a amostra desta pesquisa foi realizada tendo por base um conjunto de palavras-chave frequentemente mencionadas em estudos da área de jogos e simulações. Para tanto, considerou-se a revisão efetuada por Bragge et al. (2010), na qual os autores listam os termos mais comuns nos títulos de artigos que tratam do assunto em questão. Assim, a partir dos nove termos citados pelos autores como sendo os mais comuns, foram selecionados os seguintes para realização da busca nas bases de dados: simulação, jogo, jogo de empresas e simulação de negócios.

Para a busca dos artigos, foram utilizados os sistemas de indexação dos sites e dos periódicos/anais que compõem o escopo deste estudo. Os termos foram lançados no mecanismo de busca no singular e no plural, e também na língua inglesa, como os termos business game e simulation game. Na sequência, realizou-se a leitura prévia dos títulos e resumos dos diversos artigos pré-selecionados, considerando o contexto em que os termos são empregados. 
Após a definição da amostra de artigos, o trabalho se concentrou no processo de construção da tipologia para classificação, o que não ocorreu de forma linear. Para o desenvolvimento de uma tipologia adequada aos propósitos da pesquisa, foi elaborado um protocolo para a análise e a codificação dos estudos selecionados, conforme descrito:

1. Extração aleatória de 15 artigos da amostra previamente selecionada;

2. Leitura e análise dos artigos, considerando particularmente os objetivos do estudo, os resultados obtidos e a respectiva metodologia, conforme explicitado pelos autores;

3. Classificação preliminar do estudo, considerando o tipo de abordagem ao fenômeno, o eixo temático, a abordagem metodológica e o método de pesquisa;

4. Extração aleatória de mais 15 artigos e repetição do procedimento de análise descrito anteriormente;

5. Revisão das classificações tendo por base as dissonâncias observadas em cada etapa de análise dos grupos de 15 artigos;

6. Atualização da tipologia inicialmente proposta;

7. Revisão dos artigos já enquadrados;

8. Repetição do ciclo de análise.

As etapas descritas foram repetidas inúmeras vezes até que todos os artigos tivessem sido analisados e adequadamente enquadrados conforme a tipologia estabelecida. O tratamento dos dados derivados desse processo foi efetuado com o auxílio de planilha Excel para a organização e a consolidação dos resultados.

Por fim, é importante salientar que a classificação da amostra de artigos (quanto à abordagem ao fenômeno e aos eixos temáticos) é resultante de exaustivo processo de leitura e codificação realizados ao longo do desenvolvimento deste estudo. Por outro lado, para o enquadramento quanto à abordagem metodológica, considerou-se, sem qualquer intervenção, a classificação explicitada pelos autores dos artigos analisados.

\section{Apresentação dos Resultados}

A análise das publicações na área de jogos de empresas e simulações foi dividida em três partes: (a) análise da abordagem ao fenômeno de pesquisa; (b) análise da perspectiva teórica (eixos temáticos); e (c) discussão dos elementos metodológicos.

\section{Análise da abordagem ao fenômeno}

A noção de que parte das pesquisas na área de jogos de empresas tem como objeto as dinâmicas e os simuladores em si, enquanto outra parte utiliza tais sistemas como instrumentos para investigar outros problemas de pesquisa, não é inédita. Keys e Wolfe (1990) já utilizaram tal divisão para classificação dos tipos de pesquisa na área de jogo de empresas.

A utilização de simulações para pesquisa foi tratada por Cohen e Rhenman (1961), que apontaram, ainda na década de 1960, o interesse e a possibilidade de os jogos de empresas serem utilizados no contexto de laboratórios de ciências sociais, como ferramentas de_pesquisa, ou, ainda, como ferramentas laboratoriais. Gentry et al. (1984) se referem a este tipo de investigação como um paradigma de pesquisa, aplicado em diversos campos (estudos em estratégia, liderança e decisão). Ainda nesse contexto, Sauaia (2013) introduz a terminologia Laboratório de Gestão, cujos pilares conceituais 
são: (a) simulador organizacional - elemento tangível representado pelo conjunto de regras econômicas que devem ser compreendidas sob a perspectiva mercadológica, operacional e financeira; (b) jogo de empresas - elemento intangível composto pelo processo decisório e competitivo a que os participantes são submetidos, caracterizado pela tomada de decisão sob incerteza; e (c) pesquisa teórico-empírica estudos aplicados, teóricos e empíricos conduzidos pelos participantes sobre um problema gerencial que agregue valor à empresa simulada. Nesse contexto, observa-se que os participantes assumem os papéis de gestores das empresas simuladas, e também de pesquisadores, na medida em que desenvolvem pesquisa aplicada, além de maximizar a experiência de aprendizagem.

Dessa forma, a fim de distinguir a abordagem ao fenômeno, a partir da ideia de ambiente de pesquisa proposta por Gentry et al. (1984), foram definidas para este estudo as categorias: Simulações como Ambiente de Pesquisa (SCAPE) e Simulações como Objeto de Pesquisa (SCOPE), com as seguintes definições:

1. Simulações como Ambiente de Pesquisa (SCAPE): Estudos que se utilizam de contextos gerados por meio de dinâmicas de simulação no formato business game, desenvolvidas de forma estruturada e sistemática, conforme metodologia previamente definida, com o propósito de obtenção de dados que possam ser tratados qualitativa ou quantitativamente, a fim de atender aos objetivos de uma determinada pesquisa científica. Um exemplo genérico desse tipo de pesquisa é a quaseexperimental, que utiliza a simulação como ambiente laboratorial. Nesse tipo de estudo são coletados dados acerca das decisões dos sujeitos, sob circunstâncias relativamente controladas (cenários parametrizados), com objetivo de analisar possíveis variações no comportamento do tomador de decisão. Neste exemplo, o fenômeno em estudo não é o jogo de empresas, e sim o sujeito cujo comportamento varia conforme situações reproduzidas por meio do simulador.

2. Simulações como Objeto de Pesquisa (SCOPE): Estudos que têm como foco central o fenômeno jogo de empresas abordando temáticas que abrangem desenvolvimento, aplicação e efetividade desse ferramental e/ou das dinâmicas desenvolvidas, sob uma perspectiva teórica e/ou empírica. Exemplos de estudos que adotam a simulação como objeto são aqueles que buscam descrever o processo de desenvolvimento de simuladores ou dinâmicas de jogos de empresas. Nos estudos deste tipo, o fenômeno da pesquisa é a própria simulação, sua efetividade no processo de ensino e aprendizagem, ou, ainda, a satisfação dos participantes proporcionada pela dinâmica desenvolvida.

Confusões quanto à delimitação do fenômeno ocorrem quando o desenvolvimento de um simulador se configura como uma etapa de uma pesquisa maior. Nesses casos, o objetivo geral da pesquisa define a natureza do estudo, ou seja, a simulação é um meio para se atingir um fim maior, logo, aplica-se a classificação SCAPE.

Outra situação de delimitação controversa ocorre em estudos descritivos que visam apresentar uma dinâmica de jogo de empresas com aplicação teste e posterior coleta de impressões dos participantes. Neste caso, entende-se que se trata de um estudo SCOPE em que a coleta de dados posterior sobre a dinâmica não altera o propósito da simulação, que se configura como objeto, e não ferramenta para realização de pesquisas.

Uma análise do período de 1996 a 2014 (incluindo três trabalhos precursores publicados em 1977 [2] e 1988 [1]) possibilitou identificar a diferença no volume de publicações, considerando essas duas abordagens ao fenômeno (Figura 1). 


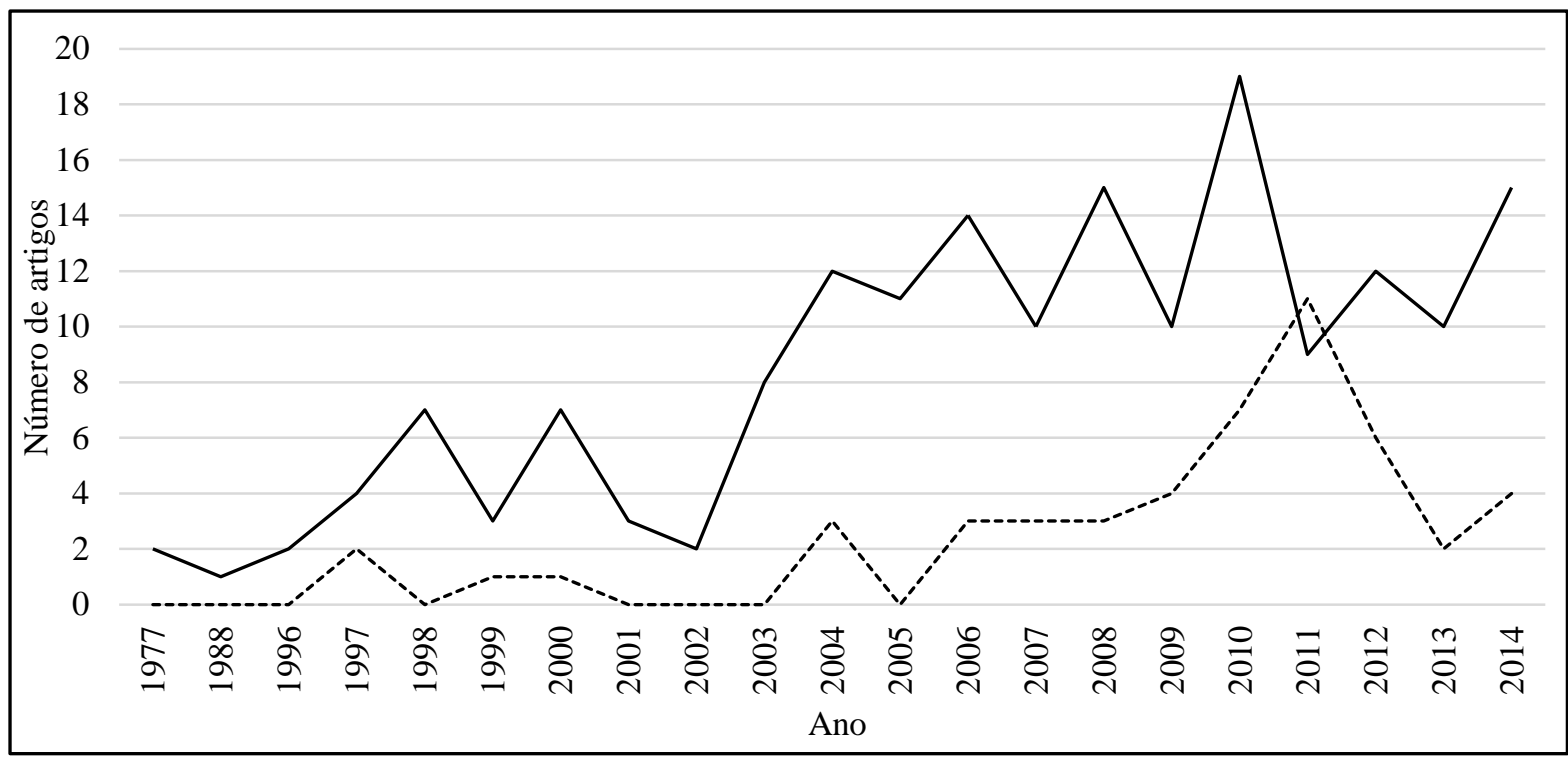

Publicações que utilizam Simulações como Ambiente de Pesquisa - SCAPE

— Publicações que utilizam Simulações como Objeto de Pesquisa - SCOPE

Figura 1. Número de Publicações ao Ano Considerando a Abordagem ao Fenômeno Fonte: Dados da pesquisa.

Apesar dos picos de publicações em 2010 (SCOPE) e 2011 (SCAPE), a produção científica nas publicações levantadas apresenta relativa estabilidade nos últimos 10 anos, com predominância dos estudos do tipo SCOPE.

O agrupamento das pesquisas conduzidas na área de jogos de empresas, além da classificação quanto à abordagem ao fenômeno, também possibilitou a investigação de questões envolvendo aspectos teóricos e metodológicos desses estudos.

\section{Perspectiva teórica: análise dos eixos temáticos}

A partir da diversidade teórica e metodológica identificada nos estudos relacionados a jogos de empresas, buscou-se desenvolver uma classificação dos eixos temáticos que agrupasse as diferentes perspectivas teóricas utilizadas. Concomitantemente, foi analisada a abordagem metodológica utilizada nas pesquisas por meio da identificação das classificações empregadas pelos autores no que se refere à abordagem metodológica, ao método e aos objetivos da pesquisa.

Considerando as perspectivas teóricas, foram identificados oito (8) eixos temáticos divididos conforme a abordagem ao fenômeno (Tabela 6). 
Tabela 6

\section{Tipologia das Áreas de Pesquisa em Jogo de Empresas e Simulações}

\begin{tabular}{lll}
\hline Abordagem ao Fenômeno & Eixo Temático & Descrição \\
\hline $\begin{array}{l}\text { Simulações como ambiente de } \\
\text { pesquisa (SCAPE) }\end{array}$ & Competências & $\begin{array}{l}\text { Estudos que abordam características e/ou } \\
\text { competências (habilidade, atitude, } \\
\text { conhecimento), individuais ou coletivas, dos } \\
\text { participantes da simulação, que podem ser }\end{array}$ \\
& & $\begin{array}{l}\text { correlacionadas com o desempenho gerencial } \\
\text { apresentado na dinâmica. }\end{array}$ \\
& & \\
\cline { 2 - 3 }
\end{tabular}

\begin{tabular}{ll}
\hline Sistema de apoio à decisão & $\begin{array}{l}\text { Estudos que tratam do desenvolvimento e/ou } \\
\text { utilização de modelos e/ou ferramentas de } \\
\text { suporte à decisão em diversos níveis } \\
\text { organizacionais. }\end{array}$
\end{tabular}

\begin{tabular}{ll}
\hline Estratégia competitiva & Estudos que adotam modelos teóricos que \\
& alicerçam investigações sobre \\
& comportamentos ou posturas estratégicas dos \\
& gestores.
\end{tabular}

Avaliação de desempenho Estudos que discutem ou propõem modelos ou indicadores de desempenho organizacional.

Processo decisório $\quad$ Estudos que abordam as diversas dimensões que envolvem o processo decisório em nível individual ou coletivo.

Simulações como objeto de Ensino-aprendizagem

\begin{tabular}{ll} 
Ensino-aprendizagem & Estudos teórico-empíricos que avaliam a \\
& efetividade dos jogos de empresas e que \\
analisam seus elementos constituintes, como & ferramenta auxiliar no processo de ensino- \\
& aprendizagem para conteúdos relacionados à \\
& gestão (marketing, produção, finanças, entre \\
& outros). \\
\hline Desenvolvimento & Estudos que envolvem iniciativas para \\
& analisar a estrutura de simuladores, modelar \\
& e/ou desenvolver e/ou complementar \\
& modelagens já existentes, integralmente ou \\
& em parte, de um jogo (computadorizado ou \\
& não) com ou sem aplicação teste. \\
\hline Estudos teórico-empíricos que tratam da \\
utilização/ disseminação/difusão dos jogos de \\
empresas e suas potencialidades \\
(mapeamentos, revisões bibliométricas, \\
estudos teóricos, análises/avaliações de \\
simuladores e jogos de empresas existentes).
\end{tabular}

Estudos teórico-empíricos que avaliam a efetividade dos jogos de empresas e que analisam seus elementos constituintes, como ferramenta auxiliar no processo de ensinoaprendizagem para conteúdos relacionados à gestão (marketing, produção, finanças, entre outros

Nota. Fonte: Elaborada pelos autores.

A descrição de cada eixo temático representou um esforço necessário para o enquadramento dos 226 artigos considerados no estudo. A análise das publicações revelou a predominância de trabalhos que tratam os jogos de empresas como objeto de estudo $(77,9 \%)$ enquanto $22,1 \%$ utilizam os jogos como instrumentos de pesquisa. Considerando a totalidade das publicações, observa-se a predominância dos estudos sobre ensino-aprendizagem $(33,2 \%)$ e os relacionados ao desenvolvimento de simuladores $(32,7 \%)$, totalizando $65,9 \%$ dos trabalhos analisados. Os estudos relativos à utilização de jogos representam $11,9 \%$ (Tabela 7 ). 
Tabela 7

Frequência de Artigos Conforme Abordagem ao Fenômeno e Eixo Temático

\begin{tabular}{llcc}
\hline Abordagem ao fenômeno & Eixos temáticos & \multicolumn{2}{c}{ Artigos } \\
\cline { 3 - 4 } & & $\mathrm{n}$ & $\%$ \\
\hline Simulações como objeto de pesquisa (SCOPE) & Ensino-aprendizagem & 75 & 33,2 \\
& Desenvolvimento & 74 & 32,7 \\
& Utilização de jogos & 27 & 11,9 \\
\cline { 2 - 4 } & Subtotal & 176 & 77,9 \\
\hline Simulações como ambiente de pesquisa (SCAPE) & Estratégia competitiva & 15 & 6,6 \\
& Competências & 11 & 4,9 \\
& Sistemas de apoio à decisão & 11 & 4,9 \\
& Processo decisório & 9 & 4,0 \\
& Avaliação de desempenho & 4 & 1,8 \\
\cline { 2 - 4 } & Subtotal & 50 & 22,1 \\
\hline & Total & 226 & 100,0 \\
\cline { 2 - 4 } & & & \\
\hline
\end{tabular}

Nota. Fonte: Dados da pesquisa.

Considerando os estudos que tratam os jogos de empresas como ambiente de pesquisa, destacamse particularmente os trabalhos com foco em estratégias competitivas, que são 15 no total. Na outra ponta, tem-se os que enfatizam processos de avaliação de desempenho, totalizando apenas quatro artigos. Os demais eixos temáticos desta categoria não apresentam expressiva variação entre si com relação ao volume de trabalhos publicados.

\section{Abordagem metodológica: classificações das pesquisas}

Além das diferentes perspectivas teóricas, a abordagem metodológica, verificada por meio das classificações das pesquisas, mostrou-se bastante variada. Tais classificações são importantes, conforme Gil (2010), por permitirem aos pesquisadores reconhecer "as semelhanças e diferenças entre as diversas modalidades de pesquisa ... e [por] conferir[em] maior racionalidade às etapas requeridas para execução" (p. 25).

Para analisar essa vertente, buscou-se verificar as classificações utilizadas pelos autores, considerando três dimensões. A primeira delas, a abordagem metodológica, foi identificada considerando uma das três vertentes explicitadas pelos autores dos estudos analisados: qualitativa, quantitativa ou métodos mistos. Em segundo lugar, foi analisado o método de pesquisa, também definido por Gil (2010) como design ou delineamento da pesquisa, que pode ser compreendido como "o planejamento da pesquisa em sua dimensão mais ampla, ... envolve os fundamentos metodológicos, a definição de objetivos, o ambiente da pesquisa e a determinação de técnicas de coleta e análise de dados" (p. 29). Assim, buscou-se identificar, dentro da abordagem escolhida pelos autores dos trabalhos analisados, a menção do método de pesquisa, como o método survey, o estudo de caso, o experimento, entre outros. Por fim, os estudos foram classificados quanto aos objetivos, considerando pesquisas exploratórias, descritivas e explicativas, além das combinações eventualmente feitas.

No que se refere à abordagem metodológica (Tabela 8), o destaque é o fato de que em um número expressivo, 165 artigos (73\%), não é mencionada a abordagem metodológica da pesquisa, sendo $72 \%$ dos artigos da categoria SCAPE e 73,3\% dos artigos da categoria SCOPE. 
Tabela 8

Frequência de Artigos de Acordo com a Abordagem Metodológica

\begin{tabular}{lllllll}
\hline Abordagem & \multicolumn{2}{l}{ SCAPE } & \multicolumn{3}{c}{ SCOPE } & \multicolumn{3}{l}{ Total } \\
\cline { 2 - 7 } & $\mathrm{n}$ & $\%$ & $\mathrm{n}$ & $\%$ & $\mathrm{~N}$ & $\%$ \\
\hline Quantitativa & 10 & 20,0 & 11 & 6,3 & 21 & 9,3 \\
Qualitativa & 1 & 2,0 & 21 & 11,9 & 22 & 9,7 \\
Qualitativa e quantitativa & 3 & 6,0 & 15 & 8,5 & 18 & 8,0 \\
\hline Subtotal & 14 & 28,0 & 47 & 26,7 & 61 & 27,0 \\
\hline Não menciona & 36 & 72,0 & 129 & 73,3 & 165 & 73,0 \\
\hline Total & 50 & 100,0 & 176 & 100,0 & 226 & 100,0 \\
\hline
\end{tabular}

Nota. Fonte: Dados da pesquisa.

Considerando o universo de artigos pesquisado, é expressiva a quantidade de estudos que não traz qualquer menção do método, 53,5\%, somando as duas categorias (Tabela 9). Apesar disso, observa-se maior número de menções do método nas pesquisas do tipo SCAPE (68\%), sendo os experimentos o método mais frequente (30\%). Na categoria SCOPE, o método mais presente é a pesquisa bibliográfica $(17 \%)$, seguido do estudo de caso $(7,4 \%)$. Considerando o total de artigos, doze estudos $(5,3 \%)$ indicaram a combinação de mais de um método.

Tabela 9

Métodos de Pesquisa de Acordo com a Abordagem ao Fenômeno

\begin{tabular}{lllllll}
\hline Método de pesquisa & SCAPE & \multicolumn{3}{c}{ SCOPE } & \multicolumn{3}{l}{ Total } \\
\cline { 2 - 7 } & $\mathrm{n}$ & $\%$ & $\mathrm{n}$ & $\%$ & $\mathrm{~N}$ & $\%$ \\
\hline Bibliográfico & - & - & 30 & 17,0 & 30 & 13,3 \\
Estudo de caso & 7 & 14,0 & 13 & 7,4 & 20 & 8,8 \\
Experimento & 15 & 30,0 & 5 & 2,8 & 20 & 8,8 \\
Quase-experimento & 5 & 10,0 & 1 & - & 6 & 2,7 \\
Survey & 1 & 2,0 & 5 & 2,8 & 6 & 2,7 \\
Documental & 2 & 4,0 & 2 & 1,1 & 4 & 1,8 \\
Pesquisação & - & - & 3 & 1,7 & 3 & 1,3 \\
Ensaio teórico & - & - & 1 & 0,6 & 1 & 0,4 \\
Fenomenológico & - & - & 1 & 0,6 & 1 & 0,4 \\
Pesquisa participante & - & - & 1 & 0,6 & 1 & 0,4 \\
Research profiling & - & - & 1 & 0,6 & 1 & 0,4 \\
Combinações & 4 & 8,0 & 8 & 4,5 & 12 & 5,3 \\
\hline Subtotal & 34 & 68,0 & 71 & 40,3 & 105 & 46,5 \\
\hline Não menciona & 16 & 32,0 & 105 & 59,7 & 121 & 53,5 \\
\hline Total & 50 & 100,0 & 176 & 100,0 & 226 & 100,0 \\
\hline
\end{tabular}

Nota. Fonte: Dados da pesquisa. 
Na maioria dos estudos do tipo SCOPE $(59,7 \%)$, não há menção do método de pesquisa. Ainda que nas pesquisas do tipo SCAPE essa frequência seja menor (32\%), tais resultados sugerem certa fragilidade metodológica da área de jogos de empresas.

O último aspecto analisado com referência à abordagem metodológica se refere ao enquadramento quanto aos objetivos da pesquisa. Nessa ótica, destacam-se as classificações como pesquisa descritiva $(12,4 \%)$, exploratória $(12,4 \%)$ e exploratória-descritiva (7,5\%). Também é expressivo o número de artigos em que não há menção de qualquer tipo de classificação. Neste caso são 147 artigos, o que corresponde a $65 \%$ do total de estudos considerados (Tabela 10).

Tabela 10

Tipos de Pesquisa Quanto aos Objetivos e Abordagem ao Fenômeno

\begin{tabular}{lllllll}
\hline \multirow{2}{*}{$\begin{array}{l}\text { Tipo de pesquisa quanto } \\
\text { aos objetivos }\end{array}$} & SCAPE & \multicolumn{3}{l}{ SCOPE } \\
\cline { 2 - 7 } & $\mathrm{n}$ & $\%$ & $\mathrm{n}$ & $\%$ & $\mathrm{n}$ & $\%$ \\
\hline Descritiva & 9 & 18,0 & 19 & 10,8 & 28 & 12,4 \\
Exploratória & 2 & 4,0 & 26 & 14,8 & 28 & 12,4 \\
Exploratória-descritiva & 4 & 8,0 & 13 & 7,4 & 17 & 7,5 \\
Explicativa & 2 & 4,0 & 2 & 1,1 & 4 & 1,8 \\
Descritiva-prescritiva & 0 & 0,0 & 1 & 0,6 & 1 & 0,4 \\
Descritiva-explicativa & 0 & 0,0 & 1 & 0,6 & 1 & 0,4 \\
\hline Subtotal & 17 & 34,0 & 62 & 35,2 & 79 & 35,0 \\
\hline Não menciona & 33 & 66,0 & 114 & 64,8 & 147 & 65,0 \\
\hline Total & 50 & 100,0 & 176 & 100,0 & 226 & 100,0 \\
\hline
\end{tabular}

Nota. Fonte: Dados da pesquisa.

A análise da frequência com que uma classificação, ou combinação de classificações, foi utilizada, revelou que 38,5\% das pesquisas que compuseram a amostra não mencionaram quaisquer das três classificações analisadas (Tabela 11).

Tabela 11

\section{Frequência da Utilização de Classificações da Pesquisa na Área de Jogos de Empresas e Simulações}

\begin{tabular}{lllllllll}
\hline Número de classificações & Tipos de classificações & \multicolumn{2}{l}{ SCAPE } & \multicolumn{2}{l}{ SCOPE } & \multicolumn{3}{c}{ Total } \\
\cline { 3 - 9 } & & $\mathrm{n}$ & $\%$ & $\mathrm{n}$ & $\%$ & $\mathrm{n}$ & $\%$ \\
\hline Uma classificação & Somente abordagem & 1 & 2,0 & 5 & 2,8 & 6 & 2,7 \\
& Somente método & 18 & 36,0 & 23 & 13,1 & 41 & 18,1 \\
& $\begin{array}{l}\text { Somente classificação quanto ao } \\
\text { objetivo }\end{array}$ & 2 & 4,0 & 7 & 4,0 & 9 & 4,0 \\
\cline { 2 - 9 } & Subtotal & 21 & 42,0 & 35 & 19,9 & 56 & 24,8 \\
\hline
\end{tabular}


Tabela 11 (continuação)

\begin{tabular}{|c|c|c|c|c|c|c|c|}
\hline \multirow[t]{2}{*}{ Número de classificações } & \multirow[t]{2}{*}{ Tipos de classificações } & \multicolumn{2}{|c|}{ SCAPE } & \multicolumn{2}{|c|}{ SCOPE } & \multicolumn{2}{|c|}{ Total } \\
\hline & & $\mathrm{n}$ & $\%$ & $\mathrm{n}$ & $\%$ & $\mathrm{n}$ & $\%$ \\
\hline \multirow[t]{4}{*}{ Duas classificações } & Abordagem + método & 5 & 10,0 & 8 & 4,5 & 13 & 5,8 \\
\hline & $\begin{array}{l}\text { Abordagem + class. quanto ao } \\
\text { objetivo }\end{array}$ & 4 & 8,0 & 15 & 8,5 & 19 & 8,4 \\
\hline & Método + objetivo & 7 & 14,0 & 21 & 11,9 & 28 & 12,4 \\
\hline & Subtotal & 16 & 32,0 & 44 & 25,0 & 60 & 26,5 \\
\hline Todas as classificações & $\begin{array}{l}\text { Abordagem + método + class. } \\
\text { quanto ao objetivo }\end{array}$ & 4 & 8,0 & 19 & 10,8 & 23 & 10,2 \\
\hline Nenhuma classificação & $\begin{array}{l}\text { Não menciona abordagem, } \\
\text { método, nem classifica o objetivo }\end{array}$ & 9 & 18,0 & 78 & 44,3 & 87 & 38,5 \\
\hline Total & & 50 & 100,0 & 176 & 100,0 & 226 & 100,0 \\
\hline
\end{tabular}

Nota. Fonte: Dados da pesquisa.

O percentual de omissões da classificação do trabalho é menor quando se consideram os estudos com enfoque nos jogos como ambiente de pesquisa (SCAPE), pois em $82 \%(42 \%+32 \%+8 \%)$ dos estudos desse tipo, uma ou mais classificações são utilizadas. Já quando se trata do SCOPE, apenas em $55,7 \%(19,9 \%+25 \%+10,8 \%)$ dos trabalhos, uma ou mais classificações são mencionadas.

Apesar de as classificações não serem determinantes para a qualidade de um estudo, representam importante indicador da consistência do processo de reflexão e da coesão da investigação desenvolvida. Classificar os estudos representa um desafio, particularmente diante das diferentes terminologias existentes, não havendo consenso entre diversos autores conforme observado por Fernandes e Gomes (2003). Apesar disso, um dos aspectos mais proeminentes se refere à definição do método. O método, ou delineamento, termo utilizado para se referir ao design da pesquisa, engloba tanto os elementos de planejamento da pesquisa como a determinação de técnicas de coleta e análise de dados (Gil, 2010).

Dessa forma, percebe-se, em particular nas pesquisas do tipo SCOPE, uma relativa desatenção no que se refere à classificação dos estudos. Apesar de diversos autores tratarem dos aspectos metodológicos descrevendo as etapas do estudo em detalhes, questiona-se até que ponto essa descrição de etapas é suficiente para a adequada sistematização da pesquisa. A omissão quanto à classificação permite que os autores se posicionem de maneira descomprometida com os pressupostos e diretrizes dos métodos de pesquisa. Assim, apesar da evolução percebida no desenvolvimento dos simuladores, alavancada em grande parte pelo avanço tecnológico, pondera-se que o amadurecimento da pesquisa na área de jogos de empresas e seu avanço dependem necessariamente de uma maior discussão acerca dos procedimentos metodológicos empregados.

\section{Conclusão}

A inspiração para o desenvolvimento do presente estudo ${ }^{(1)}$ decorre, em grande parte, da constatação da diversidade de classificação metodológica e da carência de tipologias adequadas para categorização da produção acadêmica brasileira da área de jogos de empresa.

A abordagem ao fenômeno, classificação utilizada neste estudo, representou um desafio na medida em que pressupõe a análise da intencionalidade dos autores expressa nessas duas alternativas: (a) os simuladores e jogos de empresas fazem parte do fenômeno em estudo - SCOPE; ou (b) os jogos e simuladores configuram-se em um meio para criação de um ambiente de pesquisa para manifestação 
do fenômeno a ser estudado - SCAPE. A intenção final dos pesquisadores se mostra particularmente importante, uma vez que, em alguns estudos, a construção ou adaptação de um simulador não constitui objetivo central da pesquisa, mas, sim, uma etapa metodológica importante para que se tenha acesso ao ambiente para ocorrência (ou replicação) do fenômeno de interesse na pesquisa.

Uma das principais contribuições deste estudo, ao distinguir e conceituar esses dois tipos de pesquisa (SCAPE e SCOPE), reside na possibilidade de fornecer aos pesquisadores subsídios para uma correta delimitação do que se pretende pesquisar.

A análise das publicações selecionadas revelou a predominância de pesquisas que têm os jogos de empresas como objeto de estudo (SCOPE), 77,9\%, enquanto uma parte bem menos expressiva utiliza os jogos de empresas como ambiente de pesquisa (SCAPE), representando $22,1 \%$ do total das publicações selecionadas.

Outra discussão suscitada por essa distinção é o papel dos jogos de empresas nas investigações desenvolvidas com esse foco. Nas pesquisas do tipo SCOPE, a delimitação do fenômeno possui grande sobreposição com a própria simulação ou jogo em estudo. O propósito de parte das pesquisas desse tipo, particularmente aquelas que se classificam como aplicadas, é o desenvolvimento de simuladores ou dinâmicas de jogos de empresas; no presente estudo, foram classificadas como Desenvolvimento, considerando o eixo temático. Assim, o foco dos pesquisadores reside no estudo dos simuladores e jogos de empresas em si, e em alguns de seus desdobramentos, como sua efetividade enquanto ferramenta de ensino, ou a disseminação de seu uso por professores.

Já nos estudos do tipo SCAPE, o simulador não é mais apenas um produto, mas um meio para a criação do ambiente a ser pesquisado, e deve satisfazer determinados critérios que possibilitem a geração de dados, podendo ser coletados e/ou observados considerando procedimentos metodológicos previamente selecionados. Assim, os procedimentos utilizados na simulação passam a ter uma estreita relação com a metodologia de pesquisa, entrelaçando-se e, muitas vezes, dificultando o tratamento em separado desses dois tipos de procedimentos. Um exemplo desse entrelaçamento pode ser observado em algumas pesquisas do tipo experimental ou quase-experimental que demandam ajustes no simulador, inclusões de decisões ou calibragens distintas, para que um determinado aspecto possa ser simulado.

Além de prover uma classificação quanto ao fenômeno jogo de empresas, distinguir o papel que eles exercem na pesquisa é uma das principais contribuições deste estudo. Sob uma perspectiva descritiva, percebe-se que, no Brasil, o foco predominante tem sido a pesquisa que tem os jogos de empresas como objeto de estudo, particularmente envolvendo o ensino e aprendizagem e o desenvolvimento de simuladores. Tal foco é esperado por se tratar de uma área ainda relativamente recente, porém, com os avanços tecnológicos e o amadurecimento da área, pesquisas que têm como objetivo simplesmente apresentar o processo de desenvolvimento de um jogo de empresas, em particular, perdem a relevância, especialmente com a popularização de soluções off-the-shelf e das tecnologias de programação. Nesse sentido, este estudo contribui para clarificar os contornos dos estudos que visam utilizar os jogos de empresas como ambiente de pesquisa ao prover uma clara distinção acerca das duas abordagens ao fenômeno. A menor representatividade das pesquisas tipo SCAPE $(22,1 \%)$ pode ser vista como uma oportunidade de crescimento e revigoramento desse tipo de investigação e de seu potencial de contribuição para demais áreas.

O fortalecimento das pesquisas na área de jogos de empresas passa, em parte, pela superação de fragilidades metodológicas identificadas neste estudo. Apesar de não serem determinantes para a verificação da qualidade de uma investigação, a omissão de classificações, como o método utilizado, permite levantar algumas questões metodológicas importantes, por exemplo: Diante da ausência de um método de pesquisa, a descrição dos procedimentos de coleta dos dados é suficiente para garantir a confiabilidade e a validade interna da pesquisa? Quais as contribuições e relevância das pesquisas nominadas como aplicadas, que não trazem a explicitação do método utilizado? Verifica-se, em parte dos estudos, particularmente aqueles do eixo temático Desenvolvimento, que muita atenção é dada às descrições dos jogos de empresas e dos simuladores, porém, pouco se discute sobre a validade dos dados gerados, bem como o alcance e as limitações de estudos que abordam jogos de empresas e simuladores. 
É inegável a grande quantidade de dados produzidos no decorrer de algumas sessões de um jogo de empresas, o que possibilita a aplicação do ferramental estatístico variado para a análise dos dados, entretanto, a utilização dessa gama de recursos pode inibir discussões importantes inerentes à validade dos dados coletados e à sua natureza (se são produtos de um algoritmo do jogo, ou, então, produto das decisões dos sujeitos das pesquisas). Tais questões emergem diante das informações coletadas sobre o enquadramento metodológico dos estudos, que refletem a omissão de classificações importantes, como o método da pesquisa (ausente em 53,5\% das pesquisas), do mesmo modo que questões mais singelas, como a abordagem quanto à natureza dos dados (ausente em 73\%). Observa-se que o cuidado metodológico é maior nas pesquisas do tipo SCAPE, visto que $82 \%$ apresentam pelo menos uma das classificações analisadas, enquanto nos estudos do tipo SCOPE, esse percentual cai para $55,7 \%$.

Finalmente, destaca-se que a contribuição mais relevante deste artigo é a proposição de uma tipologia para a classificação das pesquisas em jogos de empresas, e, de forma complementar, a discussão sobre as limitações metodológicas identificadas na área, que resultam em fragilidades que comprometem a qualidade de muitos estudos desenvolvidos neste campo.

É importante destacar, também, que se optou por considerar as classificações explicitadas pelos autores dos artigos analisados, em vez de se adotar uma postura analítica e interpretativa que verificasse a aderência das classificações aos procedimentos adotados para a coleta e o tratamento de dados. Apesar de poder ser considerado uma limitação, entende-se que esse procedimento conferiu maior objetividade na identificação dos aspectos metodológicos, tornando mais criterioso o enquadramento da amostra de artigos que compõem este estudo.

Considerando a delimitação, outras características dos artigos que compuseram a base analisada podem ser objeto de futuros trabalhos, como a identificação dos simuladores que são utilizados nas pesquisas do tipo SCAPE. Também se visualiza a necessidade de expansão das discussões metodológicas para além da identificação dos métodos empregados, incorporando questões envolvendo a validade e confiabilidade das pesquisas baseadas na vertente SCAPE.

\section{Nota}

\footnotetext{
${ }^{1}$ Uma versão preliminar deste artigo foi submetida ao XVII Semead - Seminários em Administração da Universidade de São Paulo em outubro de 2014.
}

\section{Referências}

Babb, E. M., Leslie, M. A., \& Van Slyke, M. D. (1966). The potential of business-gaming methods in research. The Journal of Business, 39(4), 465-472. Recuperado de http://www.jstor.org/stable/2351513. http://dx.doi.org/10.1086/294887

Barçante, L. C., \& Beltrão, K. I. (2013). 40 years of Brazilian S\&G - Analysis and perspectives. Developments in Business Simulation and Experiential Learning, 40, 314-318. Recuperado de https://journals.tdl.org/absel/index.php/absel/article/download/54/52

Bass, B. M. (1964). Business gaming for organizational research. Management Science, 10(3) 545-556. Recuperado de http://pubsonline.informs.org/doi/abs/10.1287/mnsc.10.3.545. http://dx.doi.org/10.1287/mnsc.10.3.545

Bertero, C. O. (1981). Tipologia e teoria organizacional. Revista de Administração de Empresas, 21(1), 31-38. Recuperado de http://www.scielo.br/pdf/rae/v21n1/v21n1a03.pdf. http://dx.doi.org/10.1590/S0034-75901981000100003 
Biggs, W. D. (1990). Introducing to computerized business management simulations. In J. W. Gentry (Ed.), Guide to business gaming and experiential learning (Chap. 3, pp. 23-35) East Brunswick: Nichols/GP Publishing.

Blažič, A. J., \& Blažič, B. D. J. (2015). Exploring and upgrading the educational business-game taxonomy. Journal of Educational Computing Research, 52(3), 303-340. Recuperado de http://jec.sagepub.com/content/52/3/303.abstract. http://dx.doi.org/10.1177/0735633115572959

Botelho, L. L. R., Cunha, C. C. de. A., \& Macedo, M. (2011). O método da revisão integrativa nos estudos organizacionais. Gestão e Sociedade, 5(11), 121-36. Recuperado de http://www.gestaoesociedade.org/gestaoesociedade/article/download/1220/906. http://dx.doi.org/10.21171/ges.v5i11.1220

Bragge, J., Thavikulwat, P., \& Töyli, J. (2010). Profiling 40 years of research in simulation \& gaming. Simulation \& Gaming, 41(6), 869-897. Recuperado de http://sag.sagepub.com/content/41/6/869.short. http://dx.doi.org/10.1177/1046878110387539

Cohen, K. J., \& Rhenman, E. (1961). The role of management games in education and research. Management Science, 7(2), 131-159. Recuperado de http://pubsonline.informs.org/doi/abs/10.1287/mnsc.7.2.131. http://dx.doi.org/10.1287/mnsc.7.2.131

Dieguez-Barreiro, J. H., González-Benito, J., Galende, J., \& Kondo, E. K. (2011). The use of management games in the management research agenda. Developments in Business Simulation and Experiential Learning, 38, 83-90. Recuperado de https://journals.tdl.org/absel/index.php/absel/article/download/227/193

Faria, A. J., Hutchinson, D., Wellington, W. J., \& Gold, S. (2009). Developments in business gaming: a review of the past 40 years. Simulation \& Gaming, 40(4), 464-487. Recuperado de http://sag.sagepub.com/content/40/4/464.short. http://dx.doi.org/10.1177/1046878108327585

Fernandes, L. A., \& Gomes, J. M. M. (2003). Relatórios de pesquisa nas ciências sociais: características e modalidades de investigação. ConTexto, 3(4), 1-23. Recuperado de http://seer.ufrgs.br/ConTexto/article/download/11638/6840

Gentry, J. W., Tice, T. F., Robertson, C. J., \& Gentry, M. J. (1984). Simulation gaming as a means of researching substantive issues: another look. Developments in Business Simulation \& Experiential Exercises, $11, \quad 1-5 . \quad$ Recuperado de https://journals.tdl.org/absel/index.php/absel/article/view/2201

Gil, A. C. (2010). Como elaborar projetos de pesquisa (5a ed.). São Paulo: Atlas.

Greco, M., Baldissin, N., \& Nonino, F. (2013). An exploratory taxonomy of business games. Simulation \& Gaming, 44(5), 645-682. Recuperado de http://sag.sagepub.com/content/44/5/645.short. http://dx.doi.org/10.1177/1046878113501464

Halpin, A. L. (2013). A review of the simulation research in the academy of management journal: suggestions for strengthening the research conducted by ABSEL members. Developments in Business Simulation and Experiential Learning, 40, 154-165. Recuperado de https://journals.tdl.org/absel/index.php/absel/article/download/34/32

Keys, B., \& Wolfe, J. (1990). The role of management games and simulations in education and research. Journal of Management, 16(2), 307-336. Recuperado de http://jom.sagepub.com/content/16/2/307.abstract. http://dx.doi.org/10.1177/014920639001600205 
Lacruz, A. J. (2004). Jogos de empresas: considerações teóricas. Caderno de Pesquisas em Administração, 11(4), 93-109. Recuperado de http://www.unifal.com.br/Bibliotecas/Artigos_Cientificos/JOGOS\%20DE\%20EMPRESAS\%20 CONSIDERA\%C3\%87\%C3\%95ES\%20TEORICAS.pdf

Larréché, J.-C. (1987). On simulations in business education and research. Journal of Business Research, 15(6), 559-571. Recuperado de http://www.sciencedirect.com/science/article/pii/0148296387900397. http://dx.doi.org/10.1016/0148-2963(87)90039-7

Motta, G., Melo, D. R. A., Oliveira, M. A., Quintella, R. H., \& Garcia, P. A. A. (2011, setembro). O perfil da pesquisa acadêmica sobre jogos de empresas entre 2001 e 2010. Anais do Encontro Nacional da Associação Nacional de Pós-Graduação e Pesquisa em Administração, Rio de Janeiro, RJ, Brasil, 35. Recuperado de http://www.anpad.org.br/admin/pdf/EPQ1318.pdf

Motta, G., Melo, D. R. A., \& Paixão, R. B. (2012). O jogo de empresas no processo de aprendizagem em administração: o discurso coletivo de alunos. Revista de Administração Contemporânea, 16(3), 342-359. Recuperado de http://www.scielo.br/scielo.php?pid=S141565552012000300002\&script=sci_arttext. http://dx.doi.org/10.1590/S1415-65552012000300002

Oliveira, M. A., Sauaia, A. C. A., Garcia, P. A. A., \& Moreira, J. S. M. (2010, setembro). Relação entre conhecimento e desempenho gerencial: análise do aprendizado dos participantes de um jogo de empresas. Anais do Encontro Nacional da Associação Nacional de Pós-Graduação e Pesquisa em Administração, Rio de Janeiro, RJ, Brasil, 34. Recuperado de http://www.anpad.org.br/admin/pdf/epq977.pdf

Paixão, R. B., Bruni, A. L., \& Carvalho, C. V. O., Jr. (2007, agosto). Jogos de empresas na academia: aspectos conceituais e metodológicos de uma amostragem de publicações brasileiras entre 1998 e 2006. Anais do Seminário de Administração da Universidade de São Paulo, São Paulo, SP, Brasil, $10 . \quad$ Recuperado de http://www.ead.fea.usp.br/semead/10semead/sistema/resultado/trabalhospdf/442.pdf

Richardson, R. J. (2012). Pesquisa social: métodos e técnicas (3a ed.). São Paulo: Atlas.

Rosas, A. R., \& Sauaia, A. C. A. (2009). Modelo conceitual de decisões no estágio de criação de um negócio: base para construção de um simulador para jogos de empresas. Revista de Administração Contemporânea, 13(4), 663-682. Recuperado de http://www.scielo.br/scielo.php?pid=S141565552009000400009\&script=sci_arttext. http://dx.doi.org/10.1590/S1415-65552009000400009

Sauaia, A. C. A. (2013). Laboratório de gestão - simulador organizacional, jogo de empresas e pesquisa aplicada (3a ed.). Barueri: Manole.

Sauvé, L., Renaud, L., Kaufman, D., \& Marquis, J. S. (2007). Distinguishing between games and simulations: a systematic review. Journal of Educational Technology \& Society, 10(3), 247-256. Recuperado de http://www.ifets.info/journals/10_3/17.pdf

Wright-Maley, C. (2015). Beyond the "Babel problem": defining simulations for the social studies. The Journal of Social Studies Research, 39(2), 63-77. http://dx.doi.org/10.1016/j.jssr.2014.10.001 


\section{Dados dos Autores}

Valdete de Oliveira Mrtvi

Rod. Celso Garcia Cid, PR 445, KM 380, 86057-970, Londrina, PR, Brasil. E-mail: mrtvi@uel.br, mrtvi@yahoo.com.br

Fernando Kaname Westphal

Rod. Celso Garcia Cid, PR 445, KM 380, 86057-970, Londrina, PR, Brasil. E-mail: fkw@uel.br; f.westphal@yahoo.com.br

Rodrigo Bandeira-de-Mello

Rua Itapeva, 474, 01332-000, São Paulo, SP, Brasil. E-mail: rodrigo.bandeira.demello@fgv.br

Paulo Roberto Feldmann

Av. Prof. Luciano Gualberto, 908, 05014-002, São Paulo, SP, Brasil. E-mail: feldmann@ usp.br; paulo.feldmann@ fia.com.br 